\title{
Computationally Efficient Inference in Large Bayesian Mixed Frequency VARs
}

\author{
Deborah Gefang* \\ University of Leicester
}

\author{
Gary Koop ${ }^{\dagger}$ \\ University of Strathclyde
}

\author{
Aubrey Poon $\ddagger$ \\ University of Strathclyde
}

\begin{abstract}
Mixed frequency Vector Autoregressions (MF-VARs) can be used to provide timely and high frequency estimates or nowcasts of variables for which data is available at a low frequency. Bayesian methods are commonly used with MF-VARs to overcome over-parameterization concerns. But Bayesian methods typically rely on computationally demanding Markov Chain Monte Carlo (MCMC) methods. In this paper, we develop Variational Bayes (VB) methods for use with MF-VARs using Dirichlet-Laplace global-local shrinkage priors. We show that these methods are accurate and computationally much more efficient than MCMC in two empirical applications involving large MF-VARs.
\end{abstract}

Keywords: Mixed Frequency, Variational inference, Vector Autoregression, Stochastic Volatility, Hierarchical Prior, Forecasting

JEL Classifications: C11, C32, C53

The Online Appendix for this paper is available at https://sites.google.com/site/garykoop/.

\footnotetext{
*Email: dg171@leicester.ac.uk

$\dagger$ Email: gary.koop@strath.ac.uk

‡Email: aubrey.poon@strath.ac.uk
} 


\section{Introduction}

Vector Autoregressions (VARs) have had great success for macroeconomic forecasting and structural analysis. Recently, there has been an upsurge of interest in mixed frequency VAR (MF-VAR) models which incorporate variables of different frequencies into a VAR. Important contributions include Carriero, Clark and Marcellino (2015), Eraker, Chiu, Foerster, Kim and Seoane (2015), Schorfheide and Song (2015), Ghysels (2016), McCracken, Owyang and Sekhposyan (2016), Brave, Butters and Justiniano (2018), Gotz and Hauzenberger (2018) and Koop, McIntyre, Mitchell and Poon (2018). A common case is when interest centers on a quarterly variable (e.g. GDP) and the researcher has available many monthly predictors. An MF-VAR allows for timely and frequent updating of forecasts or nowcasts of the quarterly variable. It also allows producing monthly estimates of the quarterly variable which can be useful in some structural contexts where the economic question of interest is at a high frequency (e.g. Cotter, Hallam and Yilmaz, 2017).

The availability of more and more data has also led to much recent interest in large VARs involving tens or even hundreds of variables. For the same reasons that large VARs are popular, one would expect large MF-VARs to be popular. Indeed, the mixed frequency nature of the MF-VAR greatly broadens the number of variables one could include. Large quarterly data sets could, in theory, be jointly modelled with large monthly data sets or even large daily financial data sets. But so far little has been done. Of the papers cited above, the largest MF-VAR is that of Brave, Butters and Justiniano (2018) which involves 7 quarterly and 30 monthly variables. The purpose of the present paper is to develop econometric methods for filling this gap.

Bayesian methods are usually used with MF-VARs and large VARs. Bayesian priors can be used to overcome the over-parameterization problems associated with both of these. Posterior and predictive densities are usually uncovered using MCMC methods and these can be computationally slow. It is possible that, for this reason, the MF-VARs cited above do not use large data sets. Using MCMC methods in an MF-VAR with 10 or 20 variables will be slow, but computationally feasible. Working with 50 or 100 variables may simply be too computationally costly, especially in the context of a recursive forecasting exercise which requires repeatedly using MCMC methods on an expanding or rolling window of data. Long computation times are also undesirable in policy circles where the goal is to release updated nowcasts or forecasts quickly as new high frequency information becomes available.

The goal of the present paper is to develop Variational Bayesian (VB) methods for large MF-VARs 
and see how well they work in practice. VB methods provide a computationally-efficient alternative to MCMC methods. A potential drawback of VB methods is that they provide only an approximation to the posterior density. In Gefang, Koop and Poon (2019), we developed VB methods for a range of large VARs and found them to be accurate and computationally efficient (see also Hajargasht and Wozniak, 2018). In VARs with 100 variables, MCMC methods took over 100 times as much computer time as comparable VB methods for a range of priors. ${ }^{1}$ The main contribution of the present paper is to extend the methods of our earlier work with large VARs to large MF-VARs. This extension is not trivial since MF-VARs are state space models and the parameter space thus includes large numbers of latent states. The prior we use for the VAR coefficients in the MF-VAR is the Dirichlet-Laplace (D-L) prior of Bhattacharya et al (2015) and a secondary contribution of this paper lies in developing VB methods for MF-VARs with this prior. Our derivations relating to the D-L prior can also be used with the VAR, thus extending Gefang, Koop and Poon (2019). We carry out two empirical applications and find VB methods to perform well in terms of estimation accuracy as well as computational efficiency.

\section{Variational Bayesian Inference}

VB methods are commonly used in many statistical fields and are increasingly being used by econometricians. Blei, Kucukelbir and McAuliffe (2017) describes the theory and practice of VB in detail. The basic idea is that a posterior, $p(\theta \mid y)$ using data $y$ for parameters $\theta$, is approximated using a simpler p.d.f. $q(\theta)$ of the form:

$$
q(\theta)=\prod_{m=1}^{M} q_{m}\left(\theta_{m}\right),
$$

where $\theta_{m}$ for $m=1, . ., M$ are the blocks of parameters which make up $\theta$. The approximation which is as close as possible in a Kullback-Liebler sense, can be shown to be

$$
q_{m}\left(\theta_{m}\right)=\exp \left[E\left(\log p\left(\theta_{m} \mid y, \theta_{-m}\right)\right)\right]
$$

where $\theta_{-m}$ denotes all parameters except those in $\theta_{m}$ and the expectation is taken over $q\left(\theta_{-m}\right)$. The VB algorithm proceeds by finding the optimal arguments in the densities $q_{m}\left(\theta_{m}\right)$ in an iterative fashion. This iterative procedure is typically much faster than MCMC. There are two ways of assessing convergence. The first is to evaluate the evidence lower bound (ELBO, see the online appendix) at

\footnotetext{
${ }^{1}$ This statement is based on 22,000 MCMC draws.
} 
each iteration. Convergence is achieved when the change in ELBO across iterations is less than some convergence criterion. Alternatively, convergence occurs when the VB estimates of parameters or states stop changing across iterations. In our empirical work, we use the former strategy for the parameters of the model (e.g. VAR coefficients and D-L prior hyperparameters) and the latter strategy with respect to the states.

\section{The Mixed Frequency VAR}

Let $\mathbf{y}_{t}=\left(\mathbf{y}_{t}^{H}, \mathbf{y}_{t}^{L}\right)^{\prime}$ be a vector of $n=n_{H}+n_{L}$ variables involving $n_{H}$ high frequency variables and $n_{L}$ low frequency variables. Time $t$ subscripts measure time at the high frequency. We write the MFVAR in a structural form which allows for equation-by-equation estimation which leads to substantial computational improvement:

$$
\mathbf{A}_{0} \mathbf{y}_{t}=\mathbf{b}_{0}+\mathbf{B}_{1} \mathbf{y}_{t-1}+\ldots+\mathbf{B}_{p} \mathbf{y}_{t-p}+\epsilon_{t}, \epsilon_{t} \sim N(0, \Sigma)
$$

for $t=1, \ldots, T$ where $\mathbf{b}_{0}$ is a $n \times 1$ vector of intercept terms, $\mathbf{B}_{i}$ is the $n \times n$ matrix of lag $i$ VAR coefficients and $\mathbf{A}_{0}$ is an $n \times n$ lower triangular matrix with ones on the diagonal.

For estimation purposes, we re-write the MF-VAR as:

$$
\mathbf{y}_{t}=\mathbf{X}_{t} \beta+\mathbf{W}_{t} \mathbf{a}+\epsilon_{t},
$$

where $\mathbf{X}_{t}=\mathrm{I}_{n} \otimes\left[1, \mathbf{y}_{t-1}^{\prime}, \ldots, \mathbf{y}_{t-p}^{\prime}\right]$ is an $n \times K$ matrix, $\beta=\operatorname{vec}\left(\left[\mathbf{b}_{0}, \mathbf{B}_{1}, \ldots, \mathbf{B}_{p}\right]^{\prime}\right)$ is $K \times 1$ vector of coefficients, a consists of the free elements of $\mathbf{A}_{0}$ stacked by rows with $\mathbf{W}_{t}$ being the $n \times m$ matrix containing the appropriate contemporaneous elements of $\mathbf{y}_{t}$. Equation (4) can be written in terms of $n$ independent equations, with the $i^{\text {th }}$ equation being:

$$
y_{i, t}=\mathbf{z}_{i, t} \theta_{i}+\epsilon_{i, t}, \epsilon_{i, t} \sim N\left(0, \sigma_{i}^{2}\right) .
$$

where $\mathbf{z}_{i, t}$ is a row vector with $k_{i}$ elements and $\theta_{i}$ is a vector containing the elements of $\beta$ and $\mathbf{a}$ pertaining to the $i^{t h}$ equation.

For the mixed frequency VAR, $\mathbf{y}_{t}^{L}$ are treated as unobserved latent variables. The relationship 
between them and the observed low frequency variables is given by

$$
\mathbf{y}_{i, t}^{L, O}=\mathbf{M}_{i, t}^{L} \Lambda_{i}^{L} \mathbf{z}_{i, t+1}
$$

where $\mathbf{y}_{i, t}^{L, O}$ denotes the observed values of the $i^{t h}$ low frequency variable for $i=1, . ., n_{L}{ }^{2}$

The form of $\mathbf{M}_{i, t}^{L}$ and $\Lambda_{i}^{L}$ in (6) depends on the frequency mis-match and the way the variables are transformed (e.g. whether the variables are logged or log differenced). Hence, we will leave them unspecified and refer the reader to the online appendix for details. But they involve setting $\mathbf{M}_{i, t}^{L}=1$ at times the low frequency variables are observed and setting it to an empty matrix at other times. When forecasting, it can also handle release delays by setting it to an empty matrix for periods before an observation is released.

For the high frequency variables, we have a similar specification:

$$
\mathbf{y}_{t}^{H, O}=\mathbf{M}_{t}^{H} \Lambda^{H} \mathbf{y}_{t}
$$

The forms for $\mathbf{M}_{t}^{H}$ and $\Lambda^{H}$ are simpler since the former is equal to 1 in all periods (unless there are release delays) and the latter is defined so as to pick out the time $t$ value of observed high frequency variable.

The mixed frequency VAR (MF-VAR) is a state space model with state equations given by (5) and measurement equations given by (6) and (7). Bayesian MCMC methods for the MF-VAR are outlined in Koop, McIntyre, Mitchell and Poon (2018).

\section{Prior Shrinkage Using The Dirichlet-Laplace Prior}

In previous work, Gefang, Koop and Poon (2019), we developed VB methods for large VARs using a number of popular global-local shrinkage priors such as the Least Absolute Shrinkage and Selection Operator (LASSO) and the stochastic search variable selection (SSVS) priors. However, recently Bhattacharya et al. (2015), has proposed an alternative, the D-L prior, which has a stronger theoretical justification than other priors (e.g. they show that the joint posterior distribution of the parameters concentrates at the optimal rate) and reduces the problem of prior hyperparameter choice to a single hyperparameter. This hyperparameter can either be set to recommended default values suggested in

\footnotetext{
${ }^{2}$ Depending on which transformation we use, the $\mathbf{z}_{i, t+1}$ in this equation may have to be augmented with some lags.
} 
Bhattacharya et al. (2015) or can be treated as unknown and estimated. The D-L prior has been used in MF-VARs in Koop, McIntyre, Mitchell and Poon (2018) using MCMC methods. In this paper we develop VB methods for use with the MF-VAR with the D-L prior which are computationally practical even in very high dimensional models.

The D-L prior is given by:

$$
\theta_{i, j} \mid \phi_{i, j}, \tau_{i} \sim D E\left(\phi_{i, j} \tau_{i}\right), \quad \phi_{i, j} \sim \operatorname{Dir}(a, \ldots, a)
$$

where $\theta_{i, j}$ is the $j^{\text {th }}$ coefficient in $\theta_{i}$ (see equation 5), $D E$ denote the double-exponential or Laplace distribution and Dir denotes the Dirichlet distribution.

The formal properties of the D-L prior are discussed in Bhattacharya et al. (2015). Here we note informally that a $D E$ prior distribution imposes $L 1$ shrinkage as is used with the Bayesian LASSO. Relative to $L 2$ shrinkage priors, this can be shown to have the desirable properties of shrinking small (unimportant) coefficients more strongly towards zero and shrinking large (important) coefficients less. But the theoretical shrinkage properties of priors based solely on $L 1$ shrinkage such as the LASSO are imperfectly understood and prior hyperparameter choice can be difficult. The addition of the Dirichlet distibution for the local shrinkage prior hyperparameters surmounts these problems. The good theoretical properties of the D-L prior (e.g. as relating to posterior concentration) have been shown in Bhattacharya et al. (2015) and it involves only a single prior hyperparameter: $a$. In the empirical work in this paper we use a common default choice and set it to $\frac{1}{k_{i}+1}$. In other words, it is inversely proportional to the number of parameters in the equation being estimated. However, it is worth noting that there are other ways of selecting $a$ or it could even be treated as an unknown parameter and estimated from the data. For instance, Zhang and Bondell (2018), reasoning that the $R^{2}$ in a regression is easier to interpret than the hyperparameter $a$, develop a method for choosing $a$ which involves eliciting a prior for $R^{2}$.

It can be shown that an equivalent way of writing D-L prior is:

$$
\theta_{i, j} \sim N\left(0, \psi_{i, j} \phi_{i, j}^{2} \tau_{i}^{2}\right), \quad \psi_{i, j} \sim \operatorname{Exp}(1 / 2)
$$

Hence, the prior for all the coefficients in equation $i$ is: $\theta_{i}$ is $N\left(\mathbf{0}, \mathbf{V}_{i}\right)$ where $\mathbf{V}_{i}=$ $\operatorname{diag}\left(\psi_{i, 1} \phi_{i, 1}^{2} \tau_{i}^{2}, \ldots, \psi_{i, k_{n}} \phi_{i, k_{i}}^{2} \tau_{i}^{2}\right)$. Writing the D-L prior as a scale mixture of Normals is convenient since 
MCMC methods can exploit the (conditional) conjugacy between the prior and the Normal likelihood.

Following Bhattacharya et al. (2015), we use a Gamma prior for $\tau_{i}$ :

$$
\tau_{i} \sim G\left(k_{i} a, 1 / 2\right) .
$$

For $\sigma_{i}^{-2}$ we use a standard Gamma prior

$$
\sigma_{i}^{-2} \sim G(\underline{s}, \underline{\nu})
$$

\section{Variational Bayes Estimation of the Mixed Frequency VAR}

\section{$5.1 \quad$ Notation}

As discussed above, we do Bayesian estimation of the MF-VAR equation by equation. For notational simplicity, we will not explicitly note this in the following definitions and derivations. That is, all the data quantities and parameters below should have $i$ subscripts in the following material. With this notational convention, the unknown parameters in each equation are $\theta=\left(\theta_{1}, \ldots, \theta_{k}\right)^{\prime}, \psi=\left(\psi_{1}, \ldots, \psi_{k}\right)^{\prime}$,

$\phi=\left(\phi_{1}, \ldots, \phi_{k}\right)^{\prime}, \tau$ and $\sigma^{2}$. The vector $\mathbf{y}=\left(\mathbf{y}_{1}, \ldots, \mathbf{y}_{T}\right)^{\prime}$ contains both observed and unobserved data values as noted above. We also use notation where $\mathbf{Z}=\left(\mathbf{Z}_{1}, \ldots, \mathbf{Z}_{T}\right)^{\prime}$.

Our goal is to obtain $q(\bullet)$, the VB optimal approximating for each parameter or state $\bullet$. We will use notation where upper bars denote the arguments of these densities. These are what are optimized across iterations in the VB algorithm. So, for instance, $\overline{\mathbf{y}}$ and $\overline{\mathbf{Z}}$ will be the dependent and explanatory variables in an equation with the VB estimates of the unobserved low frequency estimates plugged in.

We will discuss VB estimation for the parameters (conditional on the VB estimates for the states) and then estimates of the states (given VB estimates of the parameters) in the next two subsections.

\subsection{VB Estimation of the Parameters}

To obtain the VB approximating densities, we require the full conditional posteriors for all parameters in the model. In this sub-section, we condition on the states so the relevant dependent and explanatory variables in each equation are $\overline{\mathbf{y}}$ and $\overline{\mathbf{Z}}$. The full conditionals are easily available. For $\theta$ and $\sigma^{2}$ textbook sources for Bayesian results for the Normal linear regression model can be used. The others are available in Bhattacharya et al. (2015). Using these we can construct the optimal $q$ densities and compute the 
ELBOs (see the online appendix for details). These are:

5.2.1 $q(\theta)$

$$
q(\theta) \sim N(\bar{\theta}, \overline{\mathbf{V}})
$$

where

$$
\begin{gathered}
\overline{\mathbf{V}}=\left(\frac{\frac{T}{2}+\underline{\nu}}{\bar{s}} \overline{\mathbf{Z}}^{\prime} \overline{\mathbf{Z}}+\mathbf{V}^{-1}\right)^{-1} \\
\bar{\theta}=\frac{\frac{T}{2}+\underline{\nu}}{\bar{s}} \overline{\mathbf{V}} \overline{\mathbf{Z}}^{\prime} \overline{\mathbf{y}}
\end{gathered}
$$

with $\mathbf{V}^{-1}=\operatorname{diag}\left(\overline{\psi_{1}^{-1}} \overline{\phi_{1}^{-2}} \overline{\tau^{-2}}, \ldots, \overline{\psi_{k}^{-1}} \overline{\phi_{k}^{-2}} \overline{\tau^{-2}}\right)$.

$\mathbf{5 . 2 . 2} q\left(\sigma^{-2}\right)$

$$
q\left(\sigma^{-2}\right) \sim G\left(\frac{T}{2}+\underline{\nu}, \bar{s}\right)
$$

where

$$
\bar{s}=\frac{1}{2}\left[\|\overline{\mathbf{y}}-\overline{\mathbf{Z}} \bar{\theta}\|^{2}+\operatorname{tr}\left(\overline{\mathbf{Z}}^{\prime} \overline{\mathbf{Z}} \overline{\mathbf{V}}\right)\right]+\underline{s} .
$$

This leads to the following value being updated by the VB algorithm:

$$
\overline{\sigma^{-2}}=\frac{\frac{T}{2}+\underline{\nu}}{\bar{s}}
$$

$\mathbf{5 . 2 . 3} q(\tau)$

$$
q(\tau) \sim \operatorname{giG}\left(k a-k, 1, \sum_{j=1}^{k} 2 \overline{\phi_{j}^{-1}}\left[\left(\overline{\theta_{j}}\right)^{2}+{\overline{\mathbf{V}^{j j}}}^{1 / 2}\right),\right.
$$

where giG denotes the generalized inverse Gaussian distribution.

Letting $\chi=\sum_{j=1}^{k} 2 \overline{\phi_{j}^{-1}}\left[\left(\overline{\theta_{j}}\right)^{2}+\overline{\mathbf{V}^{j j}}\right]^{1 / 2}$, we obtain quantities to be iterated over in VB of

$$
\bar{\tau}=\frac{\sqrt{\chi} K_{k a-k+1}(\sqrt{\chi})}{K_{k a-k}(\sqrt{\chi})}
$$

and

$$
\overline{\tau^{2}}=(\bar{\tau})^{2}+\chi\left[\frac{K_{k a-k+2}(\sqrt{\chi})}{K_{k a-k}(\sqrt{\chi})}-\left(\frac{K_{k a-k+1}(\sqrt{\chi})}{K_{k a-k}(\sqrt{\chi})}\right)^{2}\right]
$$


where $K_{*}[\bullet]$ is the modified Bessel function of the second kind.

\section{$\mathbf{5 . 2 . 4} q\left(\psi_{j}^{-1}\right)$}

$$
q\left(\psi_{j}^{-1}\right) \sim i G\left(\sqrt{\frac{\overline{\phi_{j}^{2}} \overline{\tau^{2}}}{\left(\overline{\theta_{j}}\right)^{2}+\overline{\mathbf{V}^{j j}}}}, 1\right),
$$

where iG denotes the inverse Gaussian distribution.

Letting $\rho=\sqrt{\frac{\overline{\phi_{j}^{2}} \overline{\tau^{2}}}{\left(\overline{\theta_{j}}\right)^{2}+\mathbf{V}^{j j}}}$, we obtain the quantity to be updated by $\mathrm{VB}$ of $\overline{\psi_{j}}=1+1 / \rho$.

5.2.5 $q\left(\phi_{j}\right)$

$$
q\left(\xi_{j}\right) \sim \operatorname{giG}\left(a-1,1,2 \sqrt{\left(\overline{\theta_{j}}\right)^{2}+\overline{\mathbf{V}^{j j}}}\right)
$$

Let $\varpi=2 \sqrt{\left(\overline{\theta_{j}}\right)^{2}+\overline{\mathbf{V}^{j j}}}$, we have $\overline{\xi_{j}}=\frac{\sqrt{\varpi} K_{a}(\sqrt{\varpi})}{K_{a-1}(\sqrt{\varpi})}$, and $\operatorname{var}\left(\xi_{j}\right)=\varpi\left\{\frac{K_{a+1}(\sqrt{\varpi})}{K_{a-1}(\sqrt{\varpi})}-\left[\frac{K_{a}(\sqrt{\varpi})}{K_{a-1}(\sqrt{\varpi})}\right]^{2}\right\}$.

Scaling $\xi_{j}$, we have $\overline{\phi_{j}}=\frac{\overline{\xi_{j}}}{\sum_{j=1}^{k} \overline{\xi_{j}}}$ and $\overline{\phi_{j}^{2}}=\left(\overline{\phi_{j}}\right)^{2}+\frac{\operatorname{var}\left(\xi_{j}\right)}{\left(\sum_{j=1}^{k} \overline{\xi_{j}}\right)^{2}}$. Thus, the optimal $q$ density of $\phi_{j}$ takes the following form:

$$
q\left(\phi_{j}\right) \sim g i G\left[a-1, \sum_{j=1}^{k} \overline{\xi_{j}}, \varpi /\left(\sum_{j=1}^{k} \overline{\xi_{j}}\right)\right]
$$

\subsection{VB Estimation of the States}

The MF-VAR is a state space model and VB estimation of particular state space models has been done in several places. Koop and Korobilis (2018) followed Wang et al (2016). Wang et al (2016) does the general state space model with Koop and Korobilis (2018) adapting their methods for a particular TVP regression model with hierarchical shrinkage prior. However, the state space models in these papers differ from ours in that their states are the time-varying regression coefficients which follow random walk or autoregressive processes which are assumed to be uncorrelated with one another. In contrast, our states are the unobserved high frequency values for the low frequency variables which typically will be correlated with one another.

In our case, we use a shrinkage prior on the VAR coefficients, not on the states. Hence, the arguments in the VB approximating density for the states are simple. They can just be obtained using Kalman filtering methods with the VB estimates for all the remaining parameters replaced with their VB estimates as described in the preceding sub-section. Complete details are given in the online appendix. 


\section{Empirical Applications}

In this section, we provide evidence that VB methods work well with mixed frequency VARs in two different empirical illustrations. The first is a US macroeconomic exercise involving a single low frequency variable (quarterly GDP growth) and 50 monthly variables, thus leading to a 51 dimensional MF-VAR. The second illustration involves a different frequency mis-match (annual-quarterly) and there are many more low frequency variables than high frequency variables. In both case we produce smoothed (i.e. full sample) estimates.

\subsection{Obtaining Monthly GDP Estimates for the US}

Data is taken from the popular FRED data set, see McCracken and $\mathrm{Ng}$ (2016). Complete details of the data are provided in the online appendix. The goal is to produce monthly estimates of GDP growth. Even with our very large 51 variable MF-VAR, VB methods produce reasonable estimates very quickly (in approximately ten minutes on a good personal computer), making VB methods suitable for pseudoreal time nowcasting exercises which repeatedly estimate the model on an expanding window of data. Using MCMC methods in an MF-VAR of this dimension would take days, making them unsuitable for this purpose.

To convince the reader that our VB estimates are reasonable, Figure 1 compares them to the commonly-used monthly Brave-Butters-Kelley (BBK) estimates of monthly GDP growth produced by the Chicago Fed (see https://www.chicagofed.org/publications/bbki/index). It can be seen that they match up very closely and the correlation between them is 0.95 . 


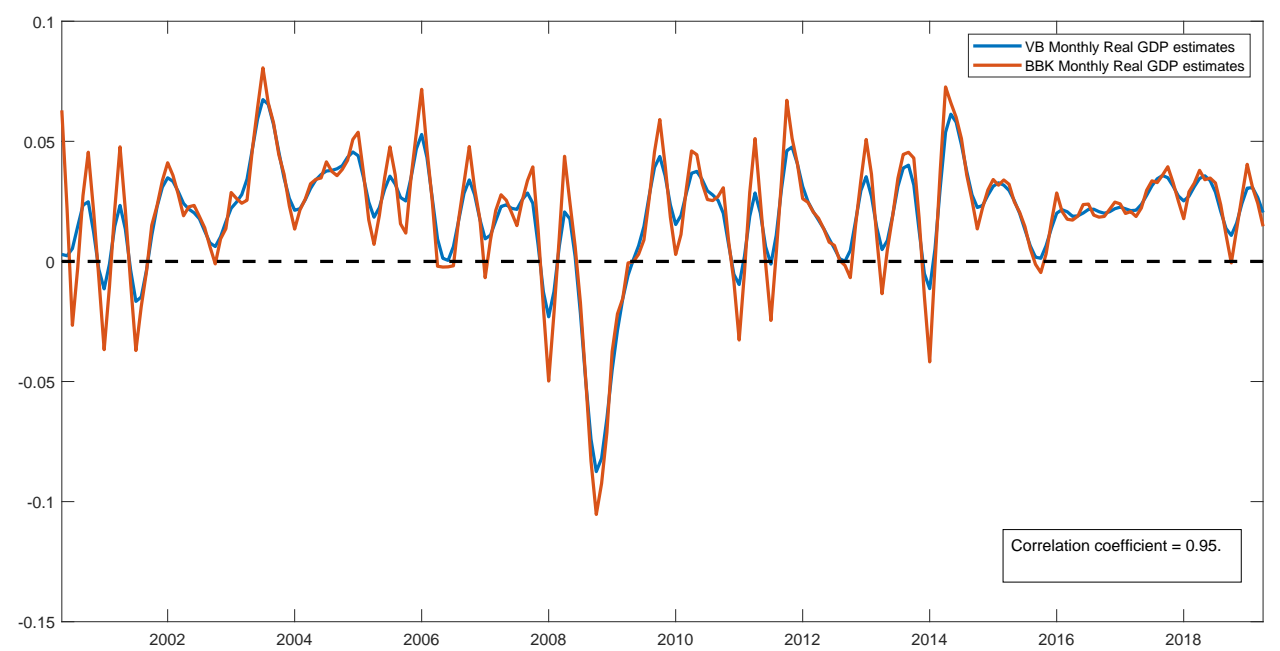

Figure 1: Monthly US GDP growth estimates: Comparison of VB vs BBK

\subsection{Historical Quarterly Estimates of Regional Growth in the UK}

In the UK, nominal Gross Value Added (GVA) is produced at the quarterly frequency for the UK as a whole, but is only produced annually for the 12 UK regions. Koop et al (2020) use a mixed frequency VAR with a Dirichlet-Laplace prior involving an annual-quarterly frequency mis-match to provide historical quarterly regional GVA estimates using MCMC methods. Here we repeat their analysis on the homoskedastic version of their model using VB methods. It is a 17 dimensional MF-VAR involving 12 annual regional GVA growth rates, quarterly UK GVA growth and four other quarterly UK predictors. Exact details of the data set and model are given in Koop et al (2020). Relative to the preceding sub-section which focussed on estimating a single quarterly variable, this is a more challenging empirical exercise due to the different frequency mis-match and the fact that high frequency estimates of many low frequency variables are required. But Koop et al (2020) found that, through the addition of an extra measurement equation which imposed the restriction that UK GVA is the sum of GVA for the regions, accurate estimation and good forecasting performance was achieved.

If repeat the MCMC-based empirical work of Koop et al (2020) using VB methods we obtain virtually identical results in a fraction of the time. Producing 20,000 draws from the MCMC algorithm took approximately five hours. Comparable VB estimation took 30 seconds. To illustrate the accuracy of VB methods, Figures 2 and 3 plot the historical estimates (posterior means) of quarterly GVA growth 
(annualized) for the regions. The high degree of similarity of MCMC and VB estimates can be seen. The correlations between VB and MCMC estimates are over 0.99 for 10 of the 12 regions. For the other two regions, the correlations are greater than 0.98 .

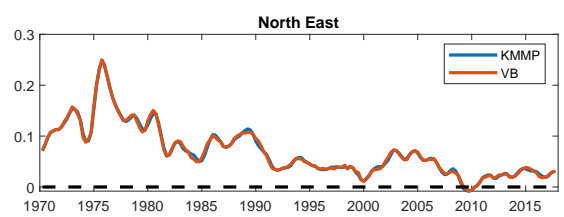

East Midlands

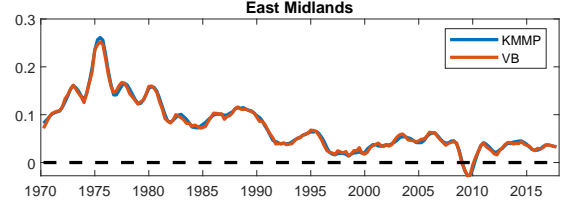

London

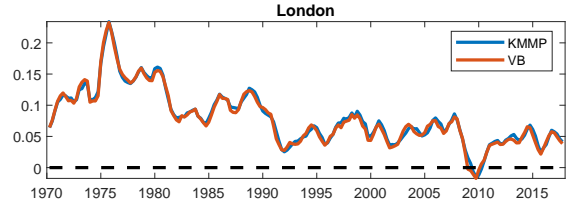

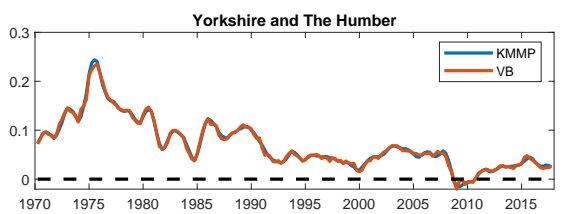

East of England

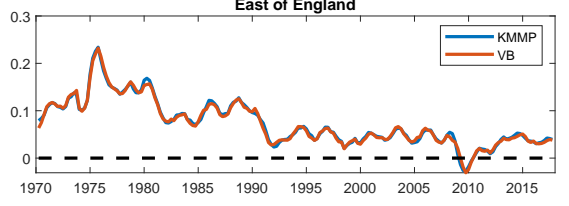

South East

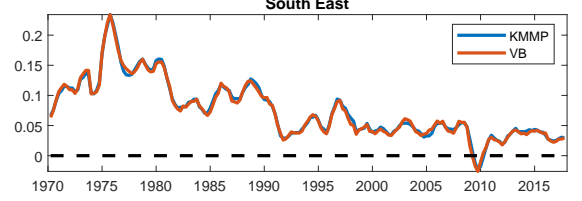

Figure 2: KMMP estimates, VB vs MCMC - Nominal GVA
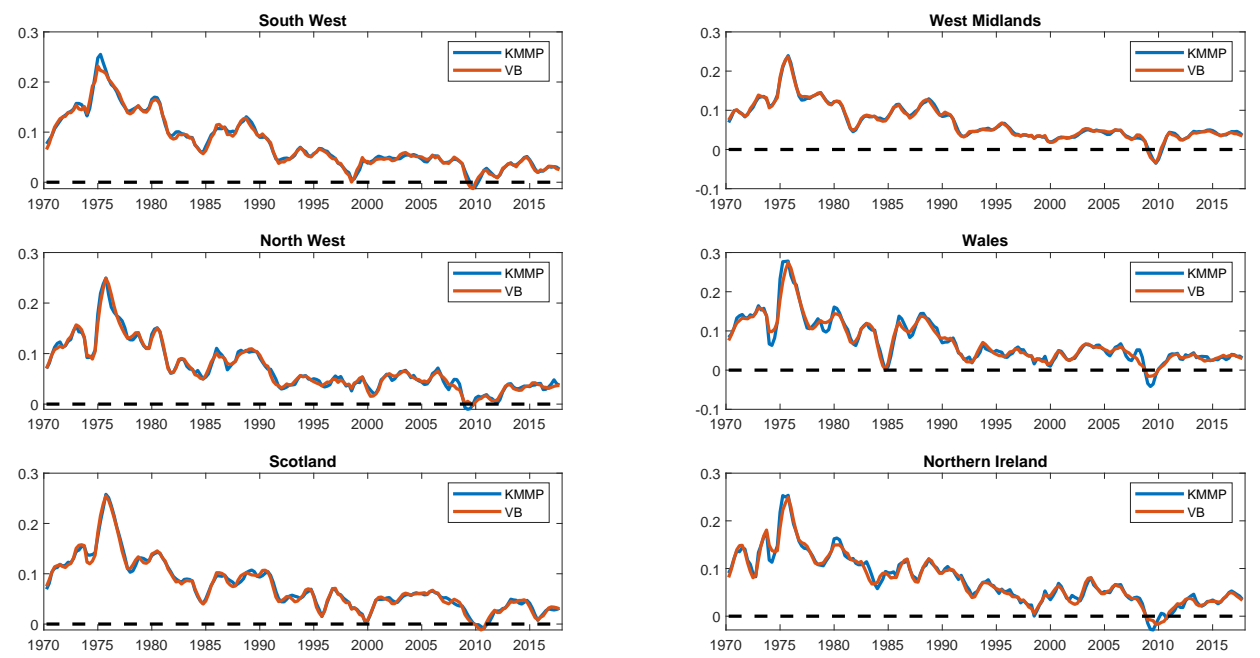

Figure 3: KMMP estimates, VB vs MCMC - Nominal GVA 


\section{Conclusions}

This paper develops VB methods for MF-VARs. These are computationally fast and scaleable and, thus, can be used with large MF-VARs where MCMC-based methods would be impracticable. In two empirical exercises, we demonstrate the accuracy and computational efficiency of our methods.

\section{References}

[1] Blei, D., Kucukelbir, A. and J. McAuliffe (2017). Variational inference: A review for statisticians. Journal of the American Statistical Association 112, 859-877.

[2] Bhattacharya, A., Pati, D., Pillai, N. S., \& Dunson, D. B. (2015). Dirichlet-Laplace priors for optimal shrinkage. Journal of the American Statistical Association, 110(512), 1479-1490.

[3] Brave, S., Butters, R. and Justiniano, A. (2018). Forecasting economic activity with mixed frequency Bayesian VARs. Federal Reserve Bank of Chicago Working Paper 2016-05 (revised July $12,2018)$.

[4] Carriero, A., Clark, T. and Marcellino, M. (2015). Realtime nowcasting with a Bayesian mixed frequency model with stochastic volatility. Journal of the Royal Statistical Society Series A 178 , $837-862$.

[5] Cotter, J., Hallam, M. and Yilmaz, K. (2017). Mixed frequency macro-financial spillovers. Koc University Working Paper 1704.

[6] Eraker, B., Chiu, C., Foerster, A., Kim, T. and Seoane, H. (2015). Bayesian mixed frequency VAR's. Journal of Financial Econometrics 13, 698-721.

[7] Gefang, D., Koop, G. and Poon, A. (2019). Variational Bayesian inference in large Vector Autoregressions with hierarchical shrinkage. Economic Statistics Centre of Excellence discussion paper 2019-07.

[8] Gotz, T. and Hauzenberger, K. (2018). Large mixed-frequency VARs with a parsimonious timevarying parameter structure. Deutsche Bundesbank Working Paper No. 40/2018.

[9] Ghysels, E. (2016). Macroeconomics and the reality of mixed frequency data. Journal of Econometrics 193, 294-314.

[10] Hajargasht, G. and Wozniak, T. (2018). Variational Bayes inference for large vector autoregressions. Manuscript.

[11] Koop, G. and Korobilis, D. (2018). Variational Bayes inference in high-dimensional time-varying parameter models. Manuscript available at https://sites.google.com/site/garykoop/research.

[12] Koop, G., McIntyre, S., Mitchell, J. and Poon, A. (2020). Regional output growth in the United Kingdom: More timely and higher frequency estimates, 1970-2017. Journal of Applied Econometrics, doi.org/10.1002/jae.2748.

[13] McCracken, M. and Ng, S. (2016). FRED-MD: A monthly database for macroeconomic research. Journal of Business and Economic Statistics 34, 574-589. 
[14] McCracken, M., Owyang, M. and Sekhposyan, T. (2016). Real-time forecasting with a large, mixed frequency Bayesian VAR, manuscript available at http://www.tateviksekhposyan.org/.

[15] Schorfheide, F. and Song, D. (2015). Real-time forecasting with a mixed-frequency VAR. Journal of Business and Economic Statistics 33(3), 366-380.

[16] Wang, H., Yu, H., Hoy, M., Dauwels, J. and Wang, H. (2016). Variational Bayesian dynamic compressive sensing, 2016 IEEE International Symposium on Information Theory.

[17] Zhang, Y. and Bondell, H. (2018). Variable selection via penalized credible regions with DirichletLaplace global-local shrinkage priors. Bayesian Analysis 13, 823-844. 